\title{
ENSINO DE ENGENHARIA DE PRODUÇÃO UTILIZANDO O MODELO PEDAGÓGICO ML-SAI
}

Ernane Rosa Martins (IFG) ernane.martins@ifg.edu.br Luís Manuel Borges Gouveia (UFP) lmbg@ufp.edu.pt

\section{Resumo}

O Modelo Pedagógico ML-SAI foi fundamentado na Sala de Aula Invertida (SAI) combinando atividades presenciais e online, personalizadas para atividades de Mobile Learning. O presente estudo foi aplicado no ensino de engenharia de produção em um curso superior do Estado de Goiás. Este trabalho de pesquisa investiga, através de um estudo de caso, como esta abordagem pode ser usada para melhorar o ensino da engenharia de produção. Os resultados da avaliação mostraram que a maioria dos alunos apreciaram a utilização do ML-SAI como uma abordagem para as aulas de engenharia de produção.

Palavras-Chaves: Sala de Aula Invertida; Engenharia de Produção; Ensino.

\section{Introdução}

Entre os conceitos utilizados neste trabalho, temos como principal a Sala de Aula Invertida (SAI), em que, o que antes era feita na sala de aula no modelo tradicional, agora é executado em casa, enquanto que as atividades que eram realizadas sozinhas pelos alunos como tarefa de casa, agora são executadas em sala de aula (BERGMANN; SAMS, 2016).

Outro conceito importante é o de aprendizagem móvel, que pode acontecer em múltiplos contextos, através de interações sociais e de conteúdo, usando dispositivos eletrônicos pessoais (CROMPTON, 2013, p. 4.). E o conceito de modelo pedagógico, que é uma reinterpretação de uma ou mais teorias de aprendizagem, que buscam promover a aprendizagem, abranger o conteúdo de ensino e desenvolver o aluno, constituídos basicamente por uma Arquitetura Pedagógica (AP) e as estratégias para aplicação desta AP, considerando geralmente aspectos, tais como: organizacionais, instrucionais, metodológicos e tecnológicos (BEHAR; PASSERINO; BERNARDI, 2007).

A principal motivação científica deste estudo foi ajudar os professores por meio de um modelo pedagógico (ML-SAI), facilitando na criação de salas de aula invertidas no ensino de engenharia de produção, com o uso ideal dos recursos humanos, tecnológicos e 
organizacionais disponíveis. Visto que o processo educacional atual apresenta em sua maioria uma abordagem tradicional de ensino, que é baseada no professor como centro do aprendizado, em que o aluno deve ouvir passivamente uma palestra, com o uso limitado de tecnologia no processo de aprendizagem, falta de colaboração e baixa comunicação dos alunos.

A ideia principal foi melhorar o ensino presencial com adoção do ensino hibrido, tornando o aprendizado mais atraente por meio das tecnologias digitais. A metodologia SAI, apresenta como vantagens os pontos fortes de ambos os ensinos, presencial e a distância. (BISSONNETTE; GAUTHIER, 2012). O principal objetivo deste trabalho é avaliar a eficiência do modelo ML-SAI em comparação com o modelo tradicional baseado em palestras. $\mathrm{O}$ restante do artigo está estruturado da seguinte forma: a seção 2 apresenta a estrutura e estratégias do ML-SAI. Na seção 3 é estabelecido o método, as técnicas e procedimentos metodológicos utilizados. Na seção 4, apresenta-se os resultados encontrados. Por fim, a seção 5 apresenta as considerações finais sobre o presente trabalho.

\section{Estrutura e estratégias do ML-SAI}

O modelo pedagógico ML-SAI fornece algumas sugestões de estratégias a professores e pesquisadores interessados em utiliza-lo, orientando estes no desenvolvimento de atividades de m-learning. A AP foi reestruturada, levando em consideração os conceitos da Sala de Aula Invertida, os aspectos relacionados a utilização dos dispositivos móveis e os estudos exploratórios preliminares realizados durante as pesquisas realizadas para sua construção. 


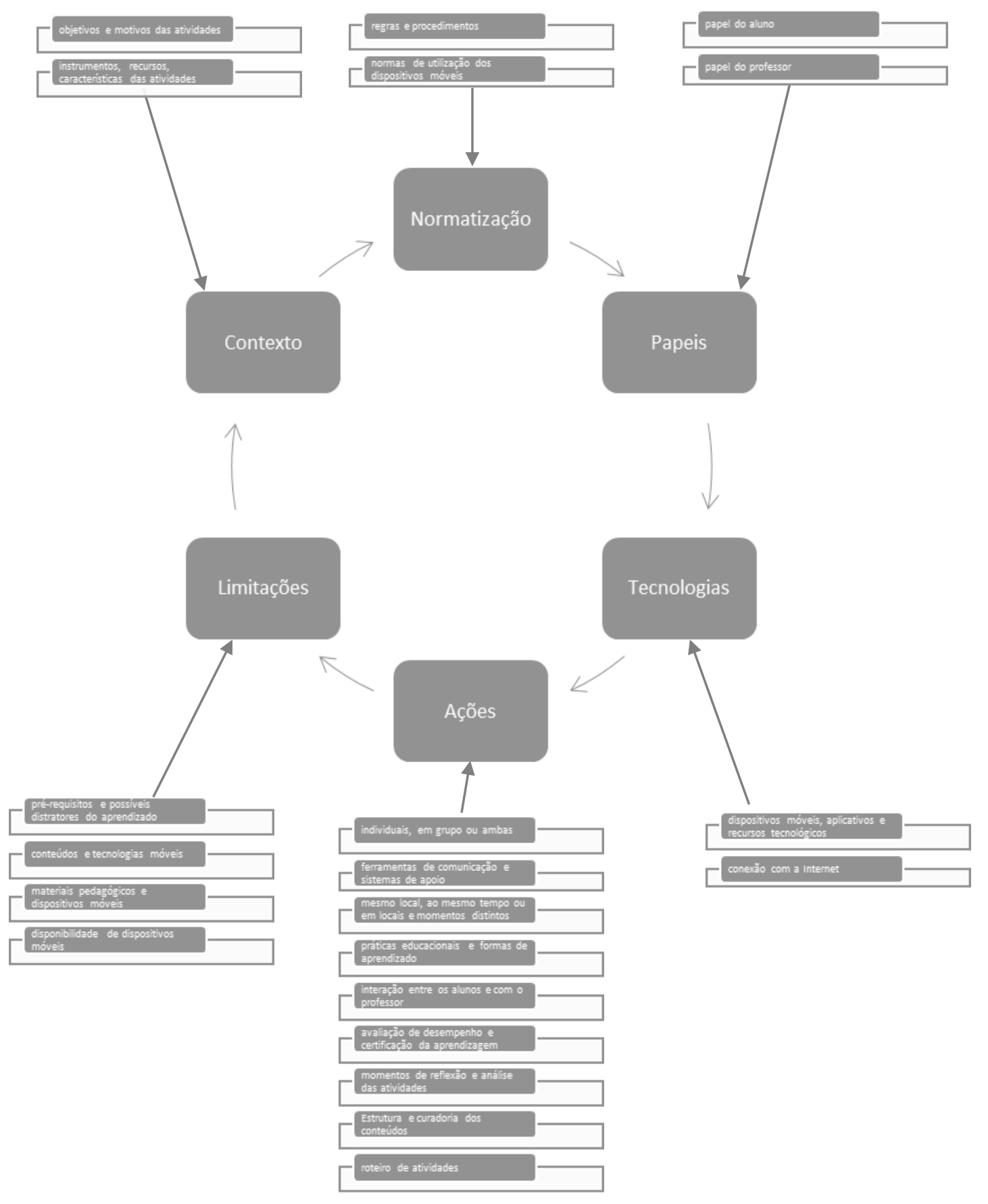

Fonte: Martins e Gouveia (2019a)

A AP foi definida em seis aspectos: contexto, normatização, papeis, tecnologias, ações e limitações, conforme ilustrado na Figura 1. A AP e as estratégias para a Aplicação da AP são detalhadas na Tabela 1 .

Tabela 1 - Detalhamento do Modelo pedagógico: ML-SAI 


\section{Arquitetura}

\section{Estratégias para a Aplicação da AP}

\section{Pedagógica}

(AP)

\begin{tabular}{|c|c|}
\hline Contexto & $\begin{array}{l}\text { Definir os objetivos e motivos das atividades e ações proposta, } \\
\text { deixando-os claros para todos os envolvidos; } \\
\text { Identificar os instrumentos, recursos, características das atividades } \\
\text { e ações, dos alunos e do curso; }\end{array}$ \\
\hline Normatizaç & $\begin{array}{l}\text { Organizar regras e procedimentos para orientar as ações e } \\
\text { interações; } \\
\text { Estabelecer normas para utilização dos dispositivos móveis } \\
\text { (quando utilizar, qual a finalidade, etc.); }\end{array}$ \\
\hline Papeis & $\begin{array}{l}\text { Compreender o papel do aluno no processo de aprendizagem, suas } \\
\text { motivações, interesses e habilidades; } \\
\text { Entender o papel do professor como condutor e facilitador da } \\
\text { aprendizagem; }\end{array}$ \\
\hline Tecnologia & $\begin{array}{l}\text { Definir os dispositivos móveis, aplicativos e recursos tecnológicos } \\
\text { que serão utilizados, considerando as características físicas, } \\
\text { técnicas e funcionais dos mesmos, tais como: ambiente virtual, } \\
\text { Sílabe, Moodle, Facebook, Khan Academy, YouTube, vídeo-aula, } \\
\text { músicas, slides, fotografias, áudios, textos, entre outros, } \\
\text { estabelecendo prioridade para aplicativos livres e gratuitos; } \\
\text { Verificar a necessidade e disponibilidade de conexão com a } \\
\text { Internet; }\end{array}$ \\
\hline Ações & $\begin{array}{l}\text { Especificar se as ações serão individuais, em grupo ou ambas, se } \\
\text { estas serão comuns a todos os alunos ou diferenciadas por aluno ou } \\
\text { grupo de alunos; } \\
\text { Definir ferramentas de comunicação e sistemas de apoio para dar } \\
\text { suporte aos alunos em caso de dificuldades; } \\
\text { Definir se as ações serão realizadas em um mesmo local, ao mesmo }\end{array}$ \\
\hline
\end{tabular}


tempo ou em locais e momentos distintos;

Estabelecer práticas educacionais favoráveis ao aprendizado (situações problemas, aplicações práticas, colaborativas, autônomas, críticas, em contextos reais, pesquisas), levando em consideração os ambientes de aprendizagem (on-line, salas de aula, laboratórios) de preferência com os dispositivos móveis dos próprios alunos;

Incentivar a interação entre os alunos e com o professor, por meio do uso de dispositivos móveis, com foco no desenvolvimento da atividade proposta;

Determinar os mecanismos de avaliação de desempenho e certificação da aprendizagem, se individuais ou em equipes, de preferência continua, e disponibiliza-los para os alunos;

Estabelecer momentos de reflexão e análise das atividades realizadas, buscando colaborar na melhoria continua de novas atividades;

Estruturar os conteúdos que serão disponibilizados em ambiente virtual, para que os alunos possam acessa-los por meio de um dispositivo móvel, quando e quantas vezes quiserem, se possível com o acompanhamento das visualizações pelo professor;

Realizar uma curadoria dos conteúdos já existentes na Internet, por meio de plataformas como Khan Academy e o YouTube em busca de bons vídeos educativos, ou caso não sejam encontrados, gravar vídeos ou áudios utilizando as ferramentas que existem no próprio dispositivo móvel;

Estimular diferentes formas de aprendizado por meio de diferentes fontes de conteúdo, tais como: vídeos, áudios, imagens, textos, slides, questões, entre outras;

Elaborar um roteiro de atividades do que será feito dentro da sala de aula, de modo a otimizar o tempo em sala de aula, utilizando projetos, trabalhos ou solução de problemas, que se conectem com 
o que foi visto previamente na plataforma;

Levantar os principais pré-requisitos das atividades e possíveis distratores do aprendizado;

Identificar quais conteúdos podem ser melhor trabalhados com tecnologias móveis;

Verificar se os materiais pedagógicos podem ser utilizados em

Limitações dispositivos móveis, considerando tamanho da tela, usabilidade, capacidade de armazenamento e modelos de dispositivos diferentes;

Verificar a disponibilidade de dispositivos móveis, tomadas para recarregar as baterias dos celulares, conexão com a Internet, quando necessário, e se os aplicativos apresentam interface adequada a aprendizagem do conteúdo.

Fonte: Martins e Gouveia (2019b)

O modelo pedagógico ML-SAI visa poder se adaptar e colaborar com o desenvolvimento de diversas atividades de m-learning envolvendo diferentes conteúdos e dispositivos móveis, levando em consideração as características especificas dos alunos envolvidos.

\section{Metodologia da pesquisa}

O presente trabalho pode ser classificado como um estudo qualitativo, descritivo e exploratório, realizado segundo os fundamentos dos estudos de caso (STAKE, 2005). A pesquisa exploratória proporciona maior familiaridade com o problema, tornando explícito, construindo hipóteses, aprimorando ideias e descobertas (GIL, 1991). Neste experimento foram utilizados os recursos tecnológicos digitais: YouTube, WhatsApp, Edmodo, entre outros, como apoio para as atividades on-line. Para a coleta de dados, utilizou-se como instrumentos, a observação feita pelo pesquisador, a coleta das percepções dos alunos envolvidos, por meio do questionário on-line e ainda por meio dos registros dos alunos no ambiente virtual Edmodo. A pesquisa foi realizada de forma totalmente anônima, visando incentivar os alunos a responder espontaneamente as perguntas. Para evitar respostas forçadas, a alternativa "não sei” sempre foi deixada como uma opção possível para os alunos.

O ML-SAI foi utilizado durante o primeiro semestre de 2019, em um experimento realizado no quarto ano de estudos de um curso superior presencial de Engenharia de Produção, com a 
participação de 32 alunos, de uma instituição de ensino do Brasil. A escolha por utilizar os dispositivos móveis dos próprios alunos e o ambiente de aprendizagem on-line Edmodo, foi para promover uma maior aproximação com o que os alunos estavam habituados a utilizar nas aulas e assim facilitar a aprendizagem. O Edmodo também apresenta como característica a possibilidade de ser usado como aplicativo instalado nos smartphones dos alunos, facilitando a sua utilização nestes tipos de dispositivos pelos alunos.

Em um primeiro momento foi realizado uma reunião de orientação, buscando uma iniciação e preparação dos alunos, abordando os conceitos e o funcionamento do aprendizado invertido, e assim expondo o planejamento adotado. Sendo esta fase apresentada de forma on-line por meio de vídeo, com um momento de esclarecimentos presencial em sala de aula, buscando promover a aceitação, maximalizar o entendimento e a motivação dos alunos, a respeito da utilização do modelo pedagógico ML-SAI. Ao final desta fase os alunos propuseram livremente 8 equipes de 4 estudantes sem nenhuma intervenção do professor.

Um segundo momento consistiu na realização de atividades propostas pelo professor pelas equipes de estudos. Estes trabalhos coletivos foram estruturados na forma de síntese dos assuntos em forma de apresentação oral, permitindo a realização de perguntas e debate das questões controversas, e entrega de relatórios no formato PDF, com comentários e justificativas da solução escolhida pela equipe. A ordem das apresentações foi determinada por sorteio. O objetivo desta fase consistiu na realização de pesquisas na literatura, a estruturação de ideias, e a ampliação das habilidades de comunicação.

\section{Analise dos resultados e discussão}

Todos os alunos participantes emitiram suas respostas, assim como o professor que também apresentou uma apreciação geral sobre o modelo pedagógico ML-SAI.

Os resultados obtidos em relação a avaliação do ML-SAI como uma abordagem para o treinamento de engenheiros, foram muito positivos pela maioria dos alunos (94\%), destacando como sendo um modelo de ensino claro e motivador que ajuda o aluno a trabalhar livremente com autonomia e interação com a equipe. Perante as respostas foi observado que em geral as atividades propostas com a utilização do ML-SAI aprimorou as habilidades, principalmente em relação ao seguinte aspectos: trabalho colaborativo, gerenciamento de projetos e distribuição de tarefas em equipe, solução de problemas técnicos, produção de conteúdo e comunicação. 
Observou-se que, os alunos que foram contra a abordagem, justificaram ser devido a distribuição injusta de tarefas em suas equipes. Em relação a transferência de conhecimento, $80 \%$ dos alunos afirmaram satisfação geral em adquirir conhecimento dos próprios assuntos abordados, é notável notar que as estudantes do sexo feminino apresentaram mais confiança em relação ao conhecimento adquirido. Além disso, o professor apreciou muito bem a utilização do ML-SAI em comparação com o modo de aula tradicional. Segundo ele, apreciou ser um supervisor do aprendizado ativo dos alunos, ao em vez de dar palestras o tempo todo. Disse ainda que o modelo envolveu os alunos e desenvolveu um aprendizado colaborativo e ativo.

Para aumentar a confiabilidade, foram utilizadas as notas dos estudantes comparadas com outros anos anteriores, de modo a qualificar e comparar o desempenho dos alunos com a utilização do ML-SAI. A partir dos resultados, observou que, todos os alunos fizeram um progresso positivo e apresentaram uma melhoria em termos da pontuação das notas em cerca de $18 \%$. A pesar destes resultados não serem conclusivos pela existência de outros fatores envolvendo turmas diferentes, principalmente devido ao perfil prévio de cada turma. Embora alguns alunos tenham indicado aversão às salas de aula invertidas, o resultado geral mostrou uma melhora relativamente positiva. Ou seja, a média desta turma que adotou o ML-SAI excedeu a média global das turmas anteriores com aprendizado tradicional, com exceção de dois estudantes, que anteriormente já apresentavam dificuldades em comunicação. Portanto, ainda há necessidade de pesquisas adicionais para identificar quais fatores são importantes para melhoria de desempenho dos alunos.

\section{Considerações finais}

Neste artigo, apresentamos o modelo pedagógico ML-SAI, que aborda o fundamento da sala de aula invertida. Para esse fim, realizou-se um experimento no quarto ano de estudos de engenharia de produção. O principal objetivo deste trabalho é avaliar a eficiência do modelo ML-SAI em comparação com o modelo tradicional baseado em palestras.

Os resultados da avaliação mostraram que a maioria dos alunos apreciou o ML-SAI como abordagem para o treinamento em engenharia de produção. Além disso, o experimento confirmou a utilidade do aprendizado invertido no ensino de engenharia e também provou o impacto positivo desse modelo de ensino no desempenho dos alunos. Obteve-se um aumento geral de cerca de $18 \%$ para todos os alunos em comparação com o ano anterior, com uma melhoria mais acentuada nas pontuações de estudantes do sexo feminino em comparação aos estudantes do sexo masculino. 
Pesquisas futuras podem lançar luz sobre questões de gênero, os diferentes aspectos da avaliação do trabalho em equipe e em outros contextos relacionados a utilização do MLSAI. Esperamos ainda confirmar até que ponto é possível adotar e aplicar esta abordagem em outras áreas do ensino.

\section{REFERÊNCIAS}

BEHAR, Patricia Alejandra; PASSERINO, Liliana and BERNARDI, Maira. Modelos Pedagógicos para Educação a Distância: pressupostos teóricos para a construção de objetos de aprendizagem. RENOTE - Revista Novas Tecnologias na Educação, v. 5, n. 2, p. 1-10. 2007.

BERGMANN, Jonathan and SAMS, Aaron. Sala de aula invertida: uma metodologia ativa de aprendizagem. 1 ed. Rio de Janeiro: LTC. 2016.

BISSONNETTE, Steve and GAUTHIER, Clermont. Faire classe à l'endroit ou à l'envers (Traditional or fip teaching?). Formation et Profession, 20(1), 23 28. 2012.

CROMPTON, Helen. A historical overview of mobile learning: Toward learner-centered education. Z. L. Berge, L. Y. Muilenburg (Eds.), Handbook of mobile learning, Routledge, Florence, p. 3-14. 2013.

GIL, Antônio Carlos. Métodos e Técnicas de Pesquisa Social. São Paulo: Editora Atlas. 2008.

MARTINS, Ernane Rosa and GOUVEIA, Luís Manuel Borges. Evolução da construção de um modelo pedagógico para atividades de M-learning. Research, Society and Development, v. 8, p. 1-13. 2019a.

MARTINS, Ernane Rosa and GOUVEIA, Luís Manuel Borges. M-Learning e Sala de Aula Invertida: Construção de um Modelo Pedagógico (ML-SAI). In: Solange Aparecida de Souza Monteiro. (Org.). Inquietações e proposituras na formação docente. 1ed. Ponta Grossa, PR: Atena Editora, v. 1, p. 184-192. 2019 b.

STAKE, Robert E. Qualitative case studies. In N. K. Denzin \& Y. S. Lincoln (Orgs.), The SAGE handbook of qualitative research (3 ed., p. 443-466). London: Sage. 2005. 